\title{
Next-Generation Sequencing-Based Genetic Diagnostic Strategies of Inherited Kidney Diseases
}

\author{
Jiahui Zhang a,b Changming Zhang ${ }^{c, d}$ Erzhi Gao ${ }^{c}$ Qing Zhou ${ }^{a, b}$ \\ aLife Sciences Institute, The Key Laboratory of Biosystems Homeostasis \& Protection of Ministry of Education, \\ Zhejiang University, Hangzhou, China; 'b Liangzhu Laboratory, Zhejiang University Medical Center, Hangzhou, China; \\ 'National Clinical Research Center of Kidney Diseases, Jinling Hospital, Nanjing University School of Medicine,

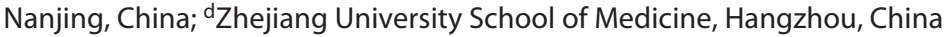

\section{Keywords}

Inherited kidney diseases · Next-generation sequencing · Variants interpretation · Genetic diagnosis

\begin{abstract}
Background: At least $10 \%$ of adults and most of the children who receive renal replacement therapy have inherited kidney diseases. These disorders substantially decrease their life quality and have a large effect on the health-care system. Multisystem complications, with typical challenges for rare disorders, including variable phenotypes and fragmented clinical and biological data, make genetic diagnosis of inherited kidney disorders difficult. In current clinical practice, genetic diagnosis is important for clinical management, estimating disease development, and applying personal treatment for patients. Summary: Inherited kidney diseases comprise hundreds of different disorders. Here, we have summarized various monogenic kidney disorders. These disorders are caused by mutations in genes coding for a wide range of proteins including receptors, channels/transporters, enzymes, transcription factors, and structural components that might also have a role in extrarenal organs (bone, eyes, brain, skin, ear, etc.). With the development of next-
\end{abstract}

karger@karger.com www.karger.com/kdd

Karger $\frac{1}{\%}$
(C) 2021 The Author(s)

Published by S. Karger AG, Basel

This is an Open Access article licensed under the Creative Commons Attribution-NonCommercial-4.0 International License (CC BY-NC) (http://www.karger.com/Services/OpenAccessLicense), applicable to the online version of the article only. Usage and distribution for commercial purposes requires written permission. generation sequencing technologies, genetic testing and analysis become more accessible, promoting our understanding of the pathophysiologic mechanisms of inherited kidney diseases. However, challenges exist in interpreting the significance of genetic variants and translating them to guide clinical managements. Alport syndrome is chosen as an example to introduce the practical application of genetic testing and diagnosis on inherited kidney diseases, considering its clinical features, genetic backgrounds, and genetic testing for making a genetic diagnosis. Key Messages: Recent advances in genomics have highlighted the complexity of Mendelian disorders, which is due to allelic heterogeneity (distinct mutations in the same gene produce distinct phenotypes), locus heterogeneity (mutations in distinct genes result in similar phenotypes), reduced penetrance, variable expressivity, modifier genes, and/or environmental factors. Implementation of precision medicine in clinical nephrology can improve the clinical diagnostic rate and treatment efficiency of kidney diseases, which requires a good understanding of genetics for nephrologists.

(C) 2021 The Author(s)

Published by S. Karger AG, Basel

Jiahui Zhang and Changming Zhang contributed equally to this work.
Correspondence to:

Qing Zhou, zhouq2@zju.edu.cn 


\section{Introduction}

Inherited kidney disorders represent a significant risk for the development of end-stage renal disease (ESRD), most of which have manifestations beginning from childhood. Recent advances in molecular genetics have made great impacts on better understanding of the molecular mechanisms of different inherited kidney diseases, some of which were not considered as genetic caused previously.

Many syndromic kidney diseases such as Alport syndrome (AS), thin basement membrane nephropathy (TBMN), autosomal dominant polycystic kidney disease (ADPKD), and a set of tubular transporter disorders are caused by pathogenic single-nucleotide variants, small frameshift insertions or deletions in the disease-causing genes. In addition, chromosomal abnormalities or copy number variants (CNVs) can cause syndromes with kidney disorders, such as Down syndrome, Turner syndrome, or Williams syndrome. The inherited kidney disorders with their genetic causes and clinical manifestations are summarized in Table 1.

In an era of precision medicine, understanding the genetic background in individuals with suspected inherited kidney diseases has important diagnostic and prognostic implications. In this review, we introduced some types of inherited kidney diseases, overviewed the application of next-generation sequencing (NGS) technologies on clinical diagnosis of inherited kidney diseases, especially in finding novel pathogenic genes, summarized the guidelines and standards for interpreting variants, and used AS as an example to specifically discuss the conditions and challenges of genetic diagnosis of inherited kidney diseases.

\section{Inherited Renal Structural Abnormality}

\section{Ciliopathies}

Ciliopathies are a class of pediatric developmental disorders that are caused by impaired function or structure of cilia $[1,2]$. Cilia is microtubule-based organelles projecting from the cytoplasmic membrane of the cell. Abnormalities of cilia can cause diseases in many organs, of which the most often affected are kidney, eye, ear, liver, lung, and brain. The diseases associated with renal ciliopathies includes PKD, nephronophthisis (NPHP), renal cystic dysplasia [2] and some multisystem ciliopathies, including Joubert syndrome and related diseases, BardetBiedl syndrome, and Meckel-Gruber syndrome [3].
Autosomal Dominant PKD

$\mathrm{ADPKD}$ is one of the most common genetic diseases among all races. Over $90 \%$ of patients with ADPKD are caused by heterozygous mutations in PKD1 or PKD2 gene, encoding the polycystin-1 or polycystin-2 proteins, respectively. Polycystin-2 regulates protein trafficking and polycystin- 1 expression. Polycystin- 1 and 2 form a heteromeric complex with fibrocystin and PKHD1, which mediates $\mathrm{Ca}^{2+}$ signaling in the primary cilium in response to the fluid flow. However, the exact mechanism on how $P K D 1 / 2$ mutations cause ADPKD is unclear [4].

Currently, there are $>2,300$ variants of $P K D 1$ and PKD2 indexed in the ADPKD mutation database (https:// pkdb.mayo.edu/cgi-bin/v2_display_mutations. cgi?apkd_mode=PROD). PKD1 mutations account for nearly $85 \%$ of affected families [5]. Genetic analysis is a helpful method for a quick and believable diagnosis. However, as the PKD1 gene is large and has a high guanine-cytosine content with a high degree of pseudohomology, obtaining a genetic diagnosis of ADPKD is challenging. Specialized screening of $P K D 1$ is required, for example, $P K D 1$-specific long-range PCR and wholegenome sequencing (WGS) can have high technical sensitivity for genetic testing. Besides, capture panel-based approaches have been developed for genetic diagnosis of ADPKD with high efficiency [6]. In addition, novel genes such as GANAB and DNAJB11 are found to cause ADP$\mathrm{KD}$ in several families [7-9].

ADPKD is usually characterized by massive enlargement of the kidneys secondary to cyst growth and development [10]. The most frequent complication is progressive renal failure which leads to ESRD at 40-59 years of age. Hypertension is very common in ADPKD and is found in most individuals with enlarged kidneys or decreased glomerular filtration rate. Other complications include acute and chronic pain, gross hematuria, cyst infection, and nephrolithiasis. The most common extrarenal manifestation is liver cysts, which present in $>90 \%$ of patients with ADPKD over 35 years of age. Other manifestations include intracranial aneurysms, development of cysts in pancreas, seminar vesicles, and ovaries [11]. $P K D 1$ and $P K D 2$ mutations have different prognostic implications. Patients with PKD2 mutations develop renal cysts, hypertension, and ESRD at a later age than patients with PKD1 mutations. Variability in disease severity within families and appearance of extrarenal manifestations have been reported [12], suggesting other genetic and environmental factors can modify the severity of this disease [13]. For example, a second mutation in $P K D 1$ or $P K D 2$ and digenic heterozygous alleles in both $P K D 1$ and 


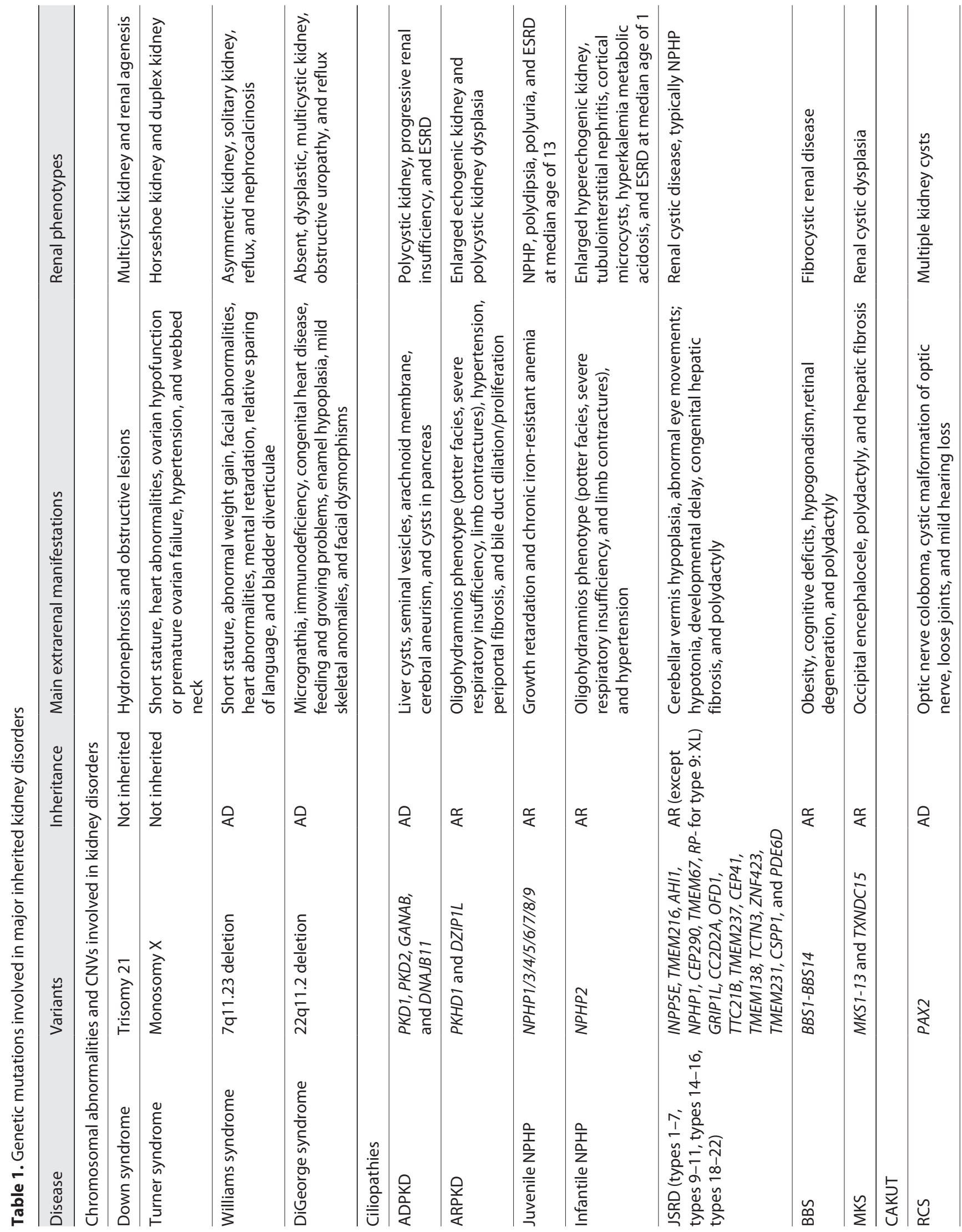




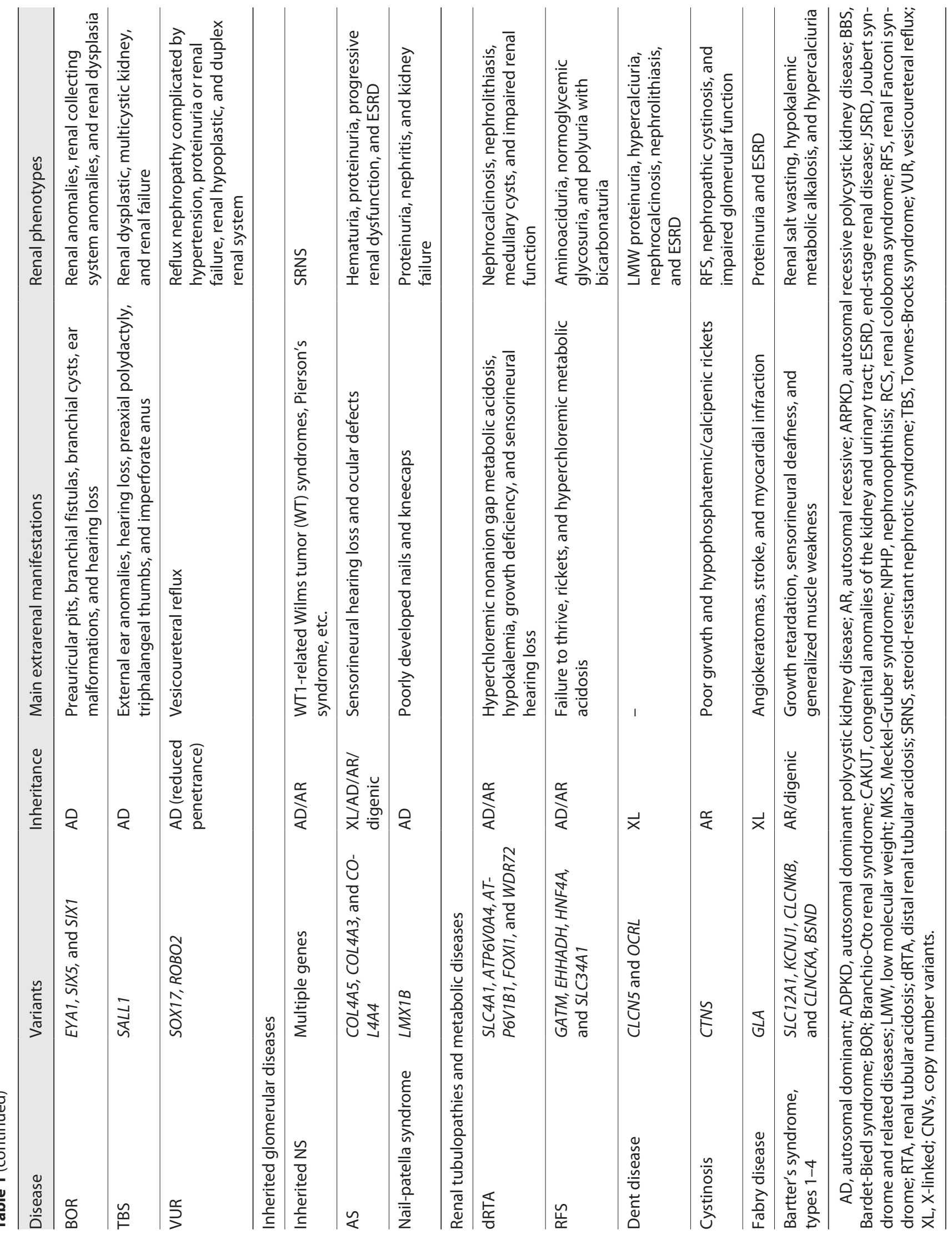


$P K D 2$ were reported as a genetic modifier associated with antenatal PKD [14]. Also, a PKD1 pathogenic allele in combination with a mutation in HNF1B may cause severe early-onset PKD [15].

\section{Autosomal Recessive PKD}

Autosomal recessive PKD (ARPKD) is rare than $\mathrm{AD}$ $\mathrm{PKD}$, caused by biallelic mutations in PKHD1 encoding the fibrocystin protein [16]. DZIP1L is another pathogenic gene of ARPKD [17]. ARPKD can be identified either prenatally or neonatally by ultrasound based on the presence of enlarged echogenic kidney. In extreme cases, severe oligohydramnios leads to Potter's phenotype, which consists of pulmonary hypoplasia, characteristic face, and deformations [18]. Molecular diagnosis is necessary to confirm a genetic diagnosis and to enable future reproductive options for a family with severe cases [19].

\section{Nephronophthisis}

NPHP is a heterogenous group of autosomal recessive cystic kidney disorders and is one of the most common reasons for ESRD in children and adolescents [1]. NPHP is characterized by reduced renal concentrating ability, chronic tubulointerstitial nephritis, cystic renal disease, and progression to ESRD before the age of 30 years old. Pathologically, NPHP presents with tubulointerstitial fibrosis, tubular dilatation, corticomedullary cyst formation, and tubular atrophy. There are 3 types of NPHP: infantile, juvenile, and adolescent form based on the age of onset for ESRD. The juvenile form is most common. To date, 8 genes (NPHP1, 3, 4, 5, 6, 7, 8, and 9) have been reported to cause the juvenile NPHP, whereas only NPHP2 is reported as a causal gene for the infantile NPHP [20].

Nearly $10-20 \%$ of NPHP have extrarenal manifestations such as retinal disease, neurological disease, liver fibrosis, and skeletal dysplasia. NPHP is also a major clinical finding in several ciliopathy syndromes, including Joubert syndrome and related diseases, Bardet-Biedl syndrome, and Meckel-Gruber syndrome [21].

Congenital Anomalies of the Kidney and Urinary Tract

Congenital anomalies of the kidney and urinary tract (CAKUT) are disorders caused by defects during embryonic kidney development. Malformations such as ureteropelvic junction obstruction, kidney agenesis, multicystic dysplastic kidneys, kidney dysplasia, kidney hypoplasia, vesicoureteral reflux, and duplex collecting system are included in the spectrum of CAKUT [22]. CAKUT can be sporadic or familial, syndromic or nonsyndromic, and the nonsyndromic form is more common. Isolated CAKUT can be associated with 500 syndromes. Syndromic CAKUT are usually associated with defects in the transcription factors that regulate critical steps in early renal development. For example, the process of the ureter budding toward the metanephric mesenchyme and mesenchyme induction/condensation.

\section{Renal Coloboma Syndrome}

Renal coloboma syndrome is an autosomal dominant condition characterized by optic nerve dysplasia and congenital kidney anomalies, usually presenting as renal hypodysplasia [23]. Histologically, kidneys exhibit reduced and enlarged glomeruli, a phenotype called oligomeganephronia. Consequences of the renal hypodysplasia include hypertension, proteinuria, and renal insufficiency that frequently progresses to ESRD. Renal coloboma syndrome is caused by mutations in the PAX2 gene [24], which is a homeobox transcription factor directly regulating the action of glial cell line-derived neurotrophic factor.

\section{Branchio-Oto Renal Syndrome}

Branchio-Oto renal (BOR) syndrome is an autosomal dominant genetic disorder with various combinations of abnormalities in kidney, brachial arches, and external/inner ear [25]. The kidney abnormalities differ among patients. In mild cases, the kidney may be unusually shaped. In severe cases, there may be duplication of the kidney collection system and/or failure of kidney development [26]. The genetic causes of BOR syndrome are mutations in the EYA1, SIX5, or SIX1, of which the EYA1 variants are the most common cause [27].

\section{Townes-Brocks Syndrome}

Townes-Brocks syndrome (TBS) is an autosomal dominant genetic disorder, characterized by the triad of imperforate anus, dysplastic ears, and thumb malformations. Renal impairment, including ESRD, may occur with or without structural abnormalities (mild malrotation, ectopia, horseshoe kidney, renal hypoplasia, polycystic kidneys, and vesicoureteral reflux). TBS is caused by pathogenic variants in SALL1 gene, encoding Sal-like protein 1 , a transcriptional repressor mainly involved in organogenesis [28]. Nonsense mutations are the most common form of pathogenic variants but deletion mutations have also been reported [29]. The highest expression of SALL1 occurs in the kidney, followed by mild expression in other organs such as developing brain and limbs, which are all affected in TBS. 


\section{Inherited Renal Dysfunction}

\section{Glomerular Diseases}

Inherited Nephrotic Syndrome

Inherited nephrotic syndrome (NS) is a group of disorders presenting with massive proteinuria. Clinically, NS mainly manifests as steroid-resistant nephrotic syndrome (SRNS). Focal segmental glomerulosclerosis (FSGS) are pathologically prevalent. Most cases of inherited NS present with an isolated glomerular disease, while some can be a syndromic disorder with extrarenal manifestations. Biallelic pathogenic variants in NPHS1 (encoding nephrin) or NPHS2 (encoding podocin) are the most common genetic cause in most of the inherited NS patients. More than 45 pathogenic genes have been associated with SRNS or inherited NS, of which some known podocyte genes can explain $57 \%-100 \%$ of familial and infant-onset NS [30]. There is significant phenotypic variability associated with inherited NS. Recessive mutations in NPHS1, NPHS2, LAMB2, and PLCE1 are reported to cause severe clinical features of early-onset NS and progress to ESRD, during infancy or throughout childhood, while recessive mutations in MYO1E and CD2AP cause severe childhood-onset NS and progress to ESRD later [30]. Autosomal dominant inherited NS is rare, occurring mostly in juvenile and adult cases. The most common causative gene is INF2 (up to 17\%), other genes include TRPC6 (up to 12\%) and ACTN4 (3.5\%) [31]. Among syndromic forms, WT1-associated glomerulopathy is the most frequent one, followed by Pierson's syndrome caused by biallelic pathogenic variants in $L A M B 2$. Once the clinical suspicion of inherited NS is raised, genetic testing should be performed by gene panel screening or whole-exome sequencing (WES) for possible pathogenic genes. Some inherited NS associated mutations have been proved to be viable therapeutic targets. For example, TRPC6 inhibitors is feasible for treating diseases that are associated with TRPC over activation in vivo, which can be a candidate therapy for patients with specific TRPC6 mutations [32].

\section{Alport Syndrome}

Alport syndrome (AS) is an inherited kidney disorder characterized by hematuria, proteinuria, and progressive renal failure. Some patients may have sensorineural deafness, eye abnormalities, esophageal leiomyoma, and other extrarenal manifestations [33]. The pathogenic genes of AS are COL4A3, COL4A4, and COL4A5, which encode type IV collagen $\alpha 3, \alpha 4$, and $\alpha 5$ chain, respectively. There are 3 types of AS: X-linked AS (XLAS), autosomal domi- nant AS (ADAS), and autosomal recessive AS (ARAS) [34]. XLAS is the most common type of AS, caused by pathogenic variants in COL4A5 gene on the X chromosome. ARAS, which takes second place, is caused by biallelic variants in COL4A3 or COL4A4. ADAS is the rarest form caused by a single variant in COL $4 A 3$ or COL $4 A 4$ [35]. An alternative genetic model that may apply to AS is digenic inheritance [36], where 2 pathogenic mutations occur respectively in COL4A3, COL4A4, or COL4A5. The genetic cause of AS can be complicated, in some cases, variants can appear in some podocyte genes, which are considered as modifier genes for the disease progression.

Different inheritance mode of AS leads to different symptoms. For XLAS, there is often a family history of hematuria (with or without proteinuria) or renal failure. Male patients with hemizygous mutations in COL4A5 are severely affected, whereas heterozygous female carriers have less severe manifestations. These female carriers usually have a broad spectrum of symptoms from isolated hematuria to ESRD, depending on the variants type and the degree of the $\mathrm{X}$ chromosome mosaicism. It is observed in female patients that approximately $98 \%$ of them have hematuria and $73 \%$ have both hematuria and proteinuria [37]. ARAS shows symptoms similar to those in male XLAS patients and there is no gender difference [37-39]. As for ADAS, median ages for developing proteinuria and renal insufficiency are 17 and 70 years old, respectively. Both hearing loss and eye lesion were reported to occur quite rarely for ADAS [33].

\section{Renal Tubulopathies and Metabolic Diseases}

The kidney provides homeostasis by a complex transport function mostly performed by specialized proteins distributed along the renal tubules. Pathogenic variants in the genes encoding these proteins will impair their function and have consequences on the whole organism, leading to renal tubulopathies [40]. Renal tubulopathies have high genetic and phenotypic heterogeneity [41]. Over 50 disease-causing genes have been identified for renal tubulopathies to date [42]. The phenotypes include polyuria and polydipsia, impaired growth, rickets and osteopenia, ultimately, and renal insufficiency.

\section{Renal Tubular Acidosis}

Renal tubular acidosis (RTA) is a group of transport defects in the process of bicarbonate $\left(\mathrm{HCO}^{-}\right)$reabsorption, or hydrogen ion $\left(\mathrm{H}^{+}\right)$excretion, or both, which result in systemic acidosis and hypokalemia with a normal glomerular filtration rate. RTA is classified into 4 types: type I (also called distal RTA, dRTA) is the classical form; 
type 2 (also called proximal RTA, pRTA) is caused by a failure of the proximal tubular cells to reabsorb filtered bicarbonate from the urine; type 3 is a type of combined pRTA and dRTA; type 4 has absolute hypoaldosteronism or aldosterone insensitivity, which is included in the RTA as it has a mild (normal anion gap) metabolic acidosis.

The genetic causes and mechanisms of dRTA have been extensively investigated $[43,44]$. The pathogenic molecular basis for all known dRTA is that, mutations in important proteins involved in acid-base transport lead to impaired acid excretion and dysfunction of intercalated cells in the collecting tubules. For now, ATP6V0A4 [45], ATP6V1B1, FOXI1, WDR72, and SLC4A1 have been identified as the pathogenic genes of dominant or recessive dRTA [46]. Isolated pRTA is rare, which is inherited as autosomal recessive manner with mutations in SCL4A4 encoding the $\mathrm{Na}^{+} / \mathrm{HCO}^{-}$cotransporter. It is usually a part of generalized proximal tubular dysfunction, termed Fanconi syndrome, with global dysfunction of all carriers in the proximal convoluted tubule.

\section{Renal Fanconi Syndrome}

Renal Fanconi syndrome (RFS) refers to the generalized dysfunction of the proximal tubule, onsets between infancy and childhood, resulting in defective of reabsorption of all small molecules. The classic manifestations include glucosuria, amino acid urine, low molecular weight proteinuria, bicarbonaturia, and acidosis [47]. RFS only affects the proximal tubule but not other nephron segments in its isolated form. However, RFS is usually secondary to a systemic autosomal recessive or X-linked disease such as cystinosis, Dent disease, and Lowe syndrome. A set of causal genes have been identified, including GATM, EHHADH, HNF4A, and SLC34A1 [48].

\section{Dent Disease}

Dent disease is a rare, $\mathrm{X}$-linked proximal renal tubular disorder that occurs almost exclusively in males. The most frequent signs are low molecular weight proteinuria and hypercalciuria. Other signs include nephrocalcinosis, nephrolithiasis, and slowly progressive renal failure. In most affected males, progressive kidney problems lead to ESRD in their early to mid-adulthood. Dent disease can result from loss-of-function (LoF) mutations in CLCN5, leading to Dent-1 (60\%) [49]; or OCRL, leading to Dent-2 (15\%) [50]. The remaining patients have a Dent disease phenotype with neither of these mutations. CIC-5, encoded by CLCN5, is involved in the acidification of early endosomes and protein reuptake by proximal tubular cells. Podocytes and parietal epithelial cells also express CIC-5, suggesting its active involvement in the onset of glomerular damage in Dent disease. Different pathogenic CLCN5 variants can reduce CIC-5 function at different degrees, which may cause phenotypic variability [51].

\section{Cystinosis}

Cystinosis, a rare autosomal recessive disorder, is a lysosomal storage disease characterized by the accumulation of free cystine in lysosomes, leading to intracellular crystal formation. It is caused by mutations in the CTNS gene that codes for cystinosin, the lysosomal membranespecific transporter for cystine [52]. There are 3 distinct types of cystinosis, each with slightly different symptoms: nephropathic cystinosis [53], intermediate cystinosis [54], and ocular, nonnephropathic cystinosis [55]. Infants affected by cystinosis initially exhibit RFS at 6-12 months of age and can develop to ESRD around 6-10 years of age. Cystine crystals may also present in the cornea and conjunctiva, leading to tearing and photophobia. Other extrarenal manifestations include growth retardation, hypothyroidism, hypergonadotropic hypogonadism, diabetes mellitus, a distal vacuolar myopathy, and dysphagia.

\section{Fabry Disease}

Fabry disease is a rare $\mathrm{X}$-linked lysosomal storage disorder that results from deficiency of lysosomal enzyme a-galactosidase A ( $\alpha$-gal A) encoded by the GLA gene [56]. Glycosphingolipids are components of plasma membrane that are degraded in the lysosome. Deficient activity of $\alpha$-gal A leads to accumulation of glycosphingolipids with terminal $\alpha$-galactosyl residues derived mostly from the turnover of cells in the kidneys, heart, lungs, nerve system, and skin. Fabry disease is a complex, multisystem disorder characterized by chronic pain and acroparesthesia, gastrointestinal disturbances, angiokeratomata, progressive renal impairment, cardiomyopathy, and stroke. Renal failure is a major complication of Fabry disease in most adult male patients. Diagnosis of Fabry disease can be made by measurement of plasma or leukocyte a-gal A activity, skin biopsy, or sequencing of the defective gene. Female with a pathogenic mutation in the GLA gene is necessary to confirm the diagnose of Fabry disease since these patients often have normal $\alpha$-gal A activity. Hundreds of mutations of GLA gene have been identified. Missense or nonsense mutations are the most frequent, and small/large deletions or insertions also appear. Mutations leading to complete LoF of the gene product are associated with classic forms of the disease, 


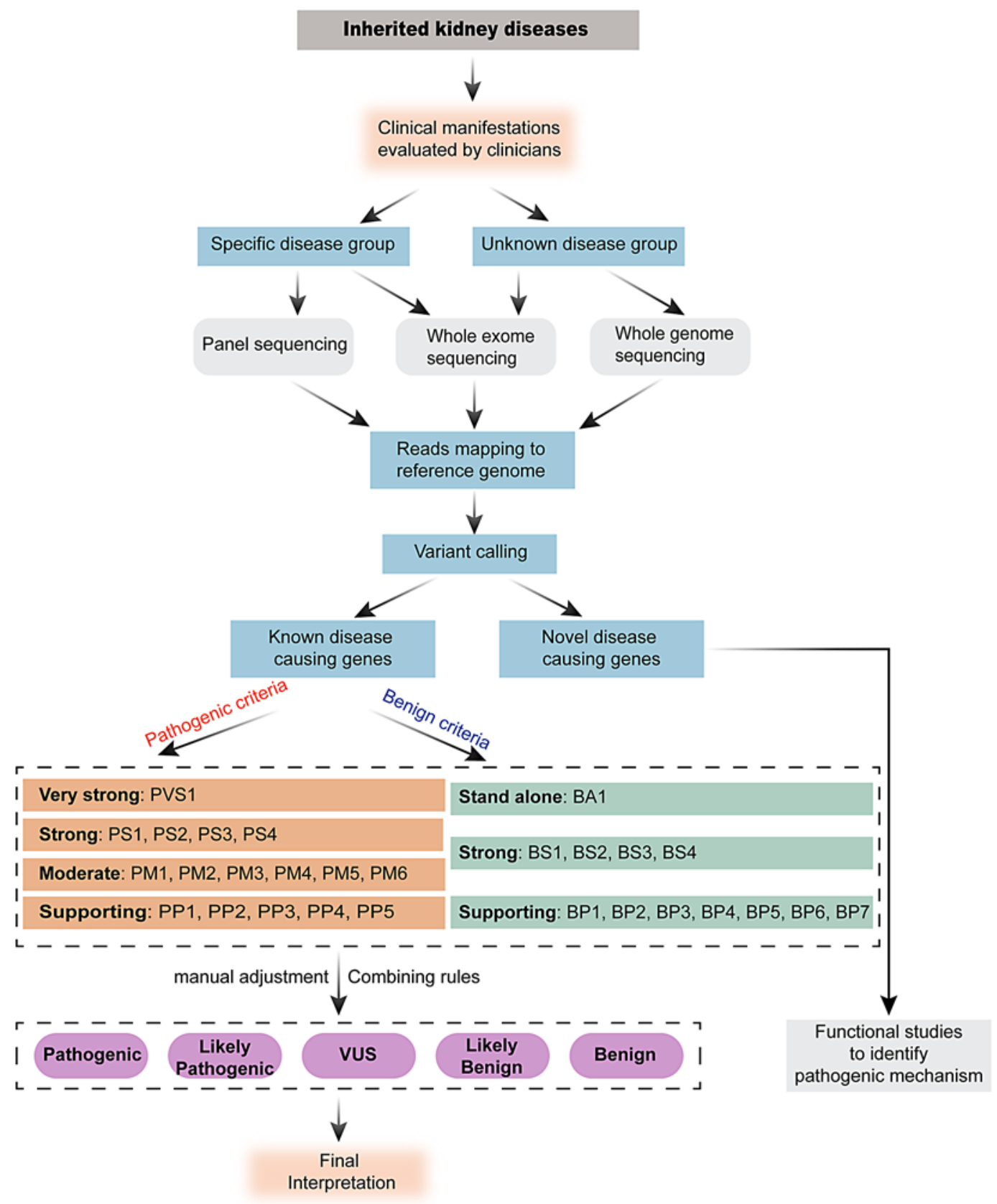

Fig. 1. Paradigm of calling and interpreting genomic variations for patients with inherited kidney diseases.

whereas mutations resulting in amino acid change might occasionally be associated with late-onset disease and milder phenotypes [57]. Recombinant enzyme replacementtherapywithintravenousagalsidase- $\alpha$ oragalsidase- $\beta$ has been applied in clinical practice since 2001 and has overall good efficacy. In addition, oral pharmacological chaperone migalastat can selectively and reversibly bind to the active center of mutant $\alpha$-gal A enzyme, so as to significantly improve the enzyme activity. Gene therapy for Fabry disease is a promising therapeutic option, whose safety is currently evaluated under several clinical studies $[58,59]$. 


\section{NGS Technologies and Genetic Diagnosis of Inherited Kidney Diseases}

\section{NGS Technologies and Their Clinical Applications}

NGS technologies refer to the deep, high-throughput, in-parallel DNA sequencing technologies. They are capable of sequencing and finding multiple candidate genes in rare disorders both for basic research and clinical practice [60]. NGS can be implemented within different applications, including WGS, WES, RNA sequencing, targeted sequencing, panel sequencing, methylation sequencing, and a set of array-based sequencing. So far, development in NGS technologies and bioinformatics analysis have greatly benefit the researches of rare diseases, as pathogenic gene identified by NGS technologies is essential for downstream clinical managements such as precise diagnosis, carrier testing, genetic counseling, and personalized therapy.

\section{Genetic Diagnosis of Inherited Kidney Diseases}

Over the last few years, diagnostics of inherited kidney diseases were improved with the accumulation of genetic knowledge on kidney pathophysiology. Our knowledge on the genetic cause of an inherited disease will not only help patients and their families to make reproductive options or other life-changing decisions but also improve our understanding of genotype-phenotype correlations, disease progression, and eventually the development of more personized therapeutics [61]. However, some patients with inherited kidney diseases have highly genetic heterogeneity that are difficult for genetic analysis. Genes that have guanine-cytosine-rich sequence or with duplicated tracts, such as COL4A3, COL4A4, COL4A5, and $P K D 1$, as well as those with CNVs, are also making difficulties for genetic analysis. A paradigm of calling and interpreting genomic variants for patients with inherited kidney diseases are shown in Figure 1.

In clinical practices, the first step for clinicians and researchers to make a genetics evaluation includes a review of the family history, personal history, and some kidney functional examination results [62]. Then, the clinicians should choose proper genetic sequencing technology for the patients based on the initial examination. If one is suspected to have a specific kind of kidney disease, applying gene panels for a targeted sequencing is an efficient option. However, if no causal variant is identified, a subsequent more comprehensive approach is in need for genetic diagnosis and future data reanalysis. The causative genetic mutation identified in the affected individual needs to be verified in his/her families by NGS technologies or Sanger sequencing.
Transforming genetic discoveries into targeted treatments is also important and challenging. For most inherited kidney diseases, treatment remains essentially unchanged and mainly supportive; however, identification of the disease causal genes and the advent of gene editing technologies [63] may provide opportunities for the future treatment of some particular kinds of inherited kidney diseases.

\section{Clinical Interpretation of Genomic Variations}

It is still a challenge to interpret the large list of genomic variation, especially those with unknown significance, which can lead to overinterpretation and even false diagnosis, unless stringent criteria are applied. To improve the accuracy of genetic diagnosis, universally accepted guidelines for variant interpretation have been developed by the American College of Medical Genetics (ACMG) [64]. It provides standards and guidelines for the interpretation of genomic variations based on evidence-based recommendations on aspects including the use of literature, database, and in silico predictors, as well as criteria for variant interpretation and reporting. These recommendations are proved to be very useful in the clinical context.

The ACMG recommends that "variant" identified in Mendelian disorders be classified into the following 5 categories: (1) pathogenic (P), (2) likely pathogenic (LP), (3) uncertain significance (VUS), (4) likely benign, or (5) benign (B). Criteria for pathogenic variants can be divided into very strong (PVS1), strong (PS1 4), moderate (PM1 6), or supporting evidence (PP1 5). Evidence for benign variants can be divided into independent (standalone, BA1), strong (BS1 4), or supporting evidence (BP1 6). For a given variation, the genetic researchers should select proper criteria based on the observed evidence.

There are several kinds of variants in the genome with different pathological significance [65]. Nonsense variants, frameshift variants, and exonic deletions/insertions (indel) are very "strong" variants, generally assumed to cause loss of function of the gene, which lead to reduced or absent protein function and nonsense-mediated RNA decay. Variants that affect splice sites may cause exon skipping and shortening or inclusion of introns, usually leading to a defected protein product.

A CNV is defined as a segment of DNA (at least $1 \mathrm{~kb}$ in size) that differs in copy number compared with a representative reference genome. It is one of the main causes of many genetic disorders. CNVs can be detected by most genomic microarrays, including array-based comparative 


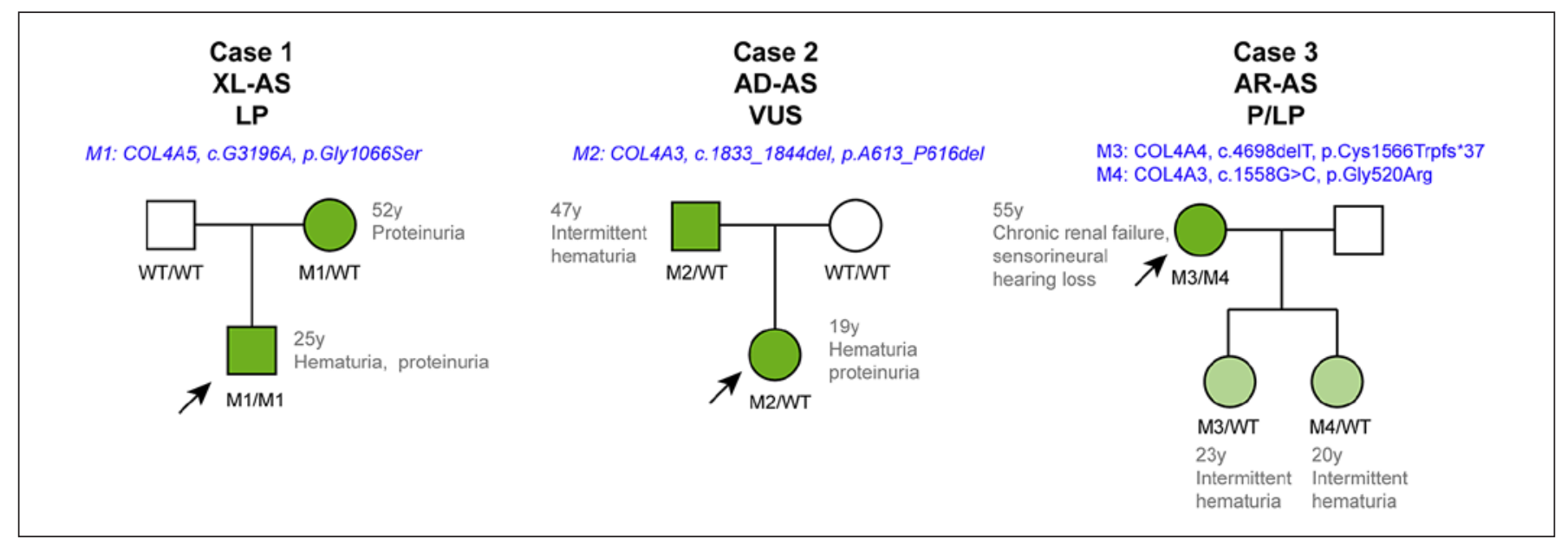

Fig. 2. Pedigree of 3 families with AS. XL-AS, X-linked Alport syndrome; LP, likely pathogenic; AD-AS, autosomal dominant Alport syndrome; VUS, uncertain significance; AR-AS, autosomal recessive Alport syndrome; P, pathogenic.

genomic hybridization (aCGH) and single-nucleotide polymorphism-based platforms. Now, NGS-based CNV analysis is increasingly used in clinical testing through genome, exome, or gene panel sequencing, but WES is less capable compared with WGS, where all regions of the genome are covered. Most CNVs are unique, requiring further investigations to determine their potential clinical significance. ACMG has provided standards and guidelines for interpretation and reporting of postnatal constitutional CNVs detected by cytogenetic microarray (e.g., chromosomal microarray) in 2011 [66]. Subsequently, ACMG and the Clinical Genome Resource (ClinGen) provided a set of technical standards for the interpretation and reporting of constitutional CNVs in 2019 [67]. In this guideline, $\mathrm{CNV}$ classification categories were changed to the 5-tier classification system recommended in the ACMG/AMP sequence variant interpretation guidelines.

\section{Application of NGS in Identifying Novel Genes of Inherited Kidney Diseases}

After applying the genetic sequencing and variant interpretation, there are still big chances that no pathogenic genes or variants are found and thus no genetic diagnosis can be made. In these cases, some novel pathogenic genes might play a role. However, the pathophysiological importance of those novel genes has not been comprehensively addressed, so it is indisputable to functionally test the identified novel variants (i.e., measurable outcomes in cell lines or animal models).

In 2011, Bredrup et al. [68] identified the WDR19 (NPHP13) gene as the pathogenic gene in patients with
NPHP using WES. This gene encodes intraflagellar transport (IFT) 144, a member in the IFT complex A that drives retrograde ciliary transport. They collected 3 unrelated families, each with different clinical manifestations: Jeune syndrome plus NPHP-like nephropathy, Sensenbrenner syndrome plus NPHP-like nephropathy, and nephropathy. WES was applied to these patients and found pathogenic variants in the shared gene WDR19. They demonstrated the ciliary pathogenesis by showing the absence of IFT144 in the cilia, as well as disrupted ciliary abundance and morphology in patient's fibroblasts. Their work identified a similar molecular cause to seemly different yet clinically overlapping disorders, which broaden the research field of inherited nephropathy.

\section{Genetic Diagnosis of AS}

Expert consensus guidelines for the genetic diagnosis of AS were published in 2018 [69]. Here we discuss the application of the genetic diagnosis of AS using 3 cases as examples, each variant was estimated based on the ACMG criteria. The pedigrees for each case are shown in Figure 2.

\section{Case 1: LP}

The proband is a 25 -year old man with hematuria (34.5 million $\mathrm{RBC} / \mathrm{mL}$ ) and proteinuria $(0.53 \mathrm{~g} / 24 \mathrm{~h})$. Genetic testing by WES identified a nonsynonymous SNV in COL4A5: c.G3196A (p.Gly1066Ser) in the proband inherited from his mother. Based on the results of immunofluorescent staining and electron microscopy observation of absence of collagen IV in the patient's kidney biopsy, this mutation shows damaging effect on the gene 
product (PS3). It is absent in population databases 1,000 Genomes Project and in extremely low frequency in Exome Aggregation Consortium (ExAC, frequency $=$ 0.00001142 ) (PM2). According to the in silico prediction using multiple lines of computational evidence, this mutation has a deleterious effect on COL4A5 protein (PP3). Besides, it has been reported as a susceptible pathogenic variant in different AS patients [70], which supports the PP5 criteria: reputable source recently reports variant as pathogenic but the evidence is not available to the laboratory to perform an independent evaluation. One strong evidence (PS3), 1 moderate evidence (PM2), and 2 supporting evidences (PP3, PP5) classify this variant as "likely pathogenic."

\section{Case 2: VUS}

The case is a 19-year old girl with persistent hematuria $(180 \mathrm{RBC} / \mathrm{mL})$ and proteinuria $(4.12 \mathrm{~g} / 24 \mathrm{~h})$. WES discovered a heterozygous nonframeshift deletion in COL4A3: c.1833_1844del, p.A613_P616del in the proband inherited from her father. This variant has not been recorded in the 1,000 Genome Database, ExAC, and Chinese Gene Mutation Database (PM2). And as a deletion mutation, it affects protein length due to in-frame deletions in a nonrepeat region (PM4). Besides, this mutation is co-segregation with disease in multiple affected family members in the AS pathogenic gene (PP1). Two moderate evidences (PM2, PM4) and 1 supporting evidence (PP1) classify this variate as "VUS."

\section{Case 3: Digenic}

Mencarelli et al. [36] reported a family with $C O L 4 A 3 /$ COL4A4 mutation combinations. In this family, individuals with 2 heterozygous mutations had more severe phenotypes (chronic renal failure, sensorineural hearing loss, and preemptive renal transplantation) than those with a single heterozygous mutation (hematuria). The first mutation was COL4A4: c.4698delT, p.Cys1566Trpfs*37. This is a frameshift variant in the pathogenic gene of AS, leading to premature stop of the transcript (PVS1). The deletion of $\mathrm{T}$ is expected to result in a truncated protein product (PM4). Besides, the mutation and the other COL4A3 mutation of the proband were inherited independently (in trans), validated by testing of his offsprings (PM3). One very strong evidence (PVS1) and 2 moderate evidences (PM3 and PM4) classify this variant as "pathogenic."

The second mutation of the proband was in COL4A3: c.G1558C, p.Gly520Arg. This mutation was classified as "likely pathogenic" because of 3 moderate evidences: (1) this is a novel missense mutation which is absent in population databases (1,000 Genomes Project, or ExAC) (PM2). (2) The missense change at this amino acid residue p.Gly520Asp has been considered to be pathogenic before (PM5). (3) This mutation and the above COL4A4 mutation of the proband were inherited independently (in trans), validated by testing of his offspring (PM3).

Genetic testing is an important criterion for AS diagnosis [71]. Several points should be noted for the genetic diagnosis of AS. To reach a genetic diagnosis of AS, genetic testing is needed for both the patient and the family, especially those without extrarenal manifestations. In individuals with suspected AS, all 3 AS genes (COL4A3, COL4A4, and COL4A5) should be examined for pathogenic variants by panel sequencing, Sanger sequencing or WES, which can identify most of the pathogenic COL4A variants. Where causative pathogenic variants cannot be demonstrated, CNV should be considered by reexamining the NGS data or with multiplex ligation-dependent probe amplification. The remaining few changes include deep intronic splicing variants or somatic mosaicism. When no pathogenic variants are found, clinical diagnosis should be reviewed, some podocyte genes such as ACTN4, NPHS2, or MYO1E and genes in which mutations produce similar clinical features to AS (e.g., FSGS, complement pathway disorders, and MYH9-related disorders) should be examined.

\section{Conclusion}

In summary, genetic diagnosis is very important for the diagnosis of hereditary kidney diseases. Proper means and appropriate samples for genetic testing should be selected based on the phenotypes in patients. After performing NGS and bioinformatics analysis, a set of candidate variants will be generated, clinical interpretation should be made in combined with patient's phenotypes, possible inheritance patterns, and consensus guidelines to interpret the pathogenicity. Incorporation of WES and WGS with traditional tools such as renal histopathology will help unravel the complex phenotypes and empower both accurate genetic diagnosis, and novel gene discovery for patients with inherited kidney diseases.

\section{Conflict of Interest Statement}

The authors have no conflicts of interest to declare. 


\section{Funding Sources}

This study did not receive any funding.

\section{Author Contributions}

J.Z. wrote the draft and prepared the figures. C.Z. provided valuable modifications into the article. E.G. and Q.Z. revised the manuscript.

\section{References}

1 Hildebrandt F, Zhou W. Nephronophthisisassociated ciliopathies. J Am Soc Nephrol. 2007 Jun;18(6): 1855-71.

2 Devlin LA, Sayer JA. Renal ciliopathies. Curr Opin Genet Dev. 2019 Jun;56:49-60.

3 Sang L, Miller JJ, Corbit KC, Giles RH, Brauer MJ, Otto EA, et al. Mapping the NPHP-JBTSMKS protein network reveals ciliopathy disease genes and pathways. Cell. 2011 May 13; 145(4):513-28.

4 Barroso-Gil M, Olinger E, Sayer JA. Molecular genetics of renal ciliopathies. Biochem Soc Trans. 2021 Jun 30;49(3):1205-20.

5 Peters DJ, Breuning MH. Autosomal dominant polycystic kidney disease: modification of disease progression. Lancet. 2001 Oct 27; 358(9291):1439-44.

6 Bergmann C, Guay-Woodford LM, Harris PC, Horie S, Peters DJM, Torres VE. Polycystic kidney disease. Nat Rev Dis Primers. 2018 Dec 6;4(1):50.

7 Porath B, Gainullin VG, Cornec-Le Gall E, Dillinger EK, Heyer CM, Hopp K, et al. Mutations in GANAB, encoding the glucosidase II $\alpha$ subunit, cause autosomal-dominant polycystic kidney and liver disease. Am J Hum Genet. 2016 Jun 2;98(6):1193-207.

8 Besse W, Choi J, Ahram D, Mane S, SannaCherchi S, Torres V, et al. A noncoding variant in GANAB explains isolated polycystic liver disease (PCLD) in a large family. Hum Mutat. 2018 Mar;39(3):378-82.

9 Cornec-Le Gall E, Olson RJ, Besse W, Heyer CM, Gainullin VG, Smith JM, et al. Monoallelic mutations to DNAJB11 cause atypical autosomal-dominant polycystic kidney disease. Am J Hum Genet. 2018 May 3;102(5): 832-44.

10 Cornec-Le Gall E, Alam A, Perrone RD. Autosomal dominant polycystic kidney disease. Lancet. 2019 Mar 2;393(10174):919-35.

11 Romao EA, Moyses Neto M, Teixeira SR, Muglia VF, Vieira-Neto OM, Dantas M. Renal and extrarenal manifestations of autosomal dominant polycystic kidney disease. Braz J Med Biol Res. 2006 Apr;39(4):533-8.

12 Idrizi A, Barbullushi M, Petrela E, Kodra S, Koroshi A, Thereska N. The influence of renal manifestations to the progression of autosomal dominant polycystic kidney disease. Hippokratia. 2009 Jul;13(3):161-4.

13 Harris PC, Rossetti S. Determinants of renal disease variability in ADPKD. Adv Chronic Kidney Dis. 2010 Mar;17(2):131-9.

14 Rossetti S, Kubly VJ, Consugar MB, Hopp K, Roy S, Horsley SW, et al. Incompletely penetrant PKD1 alleles suggest a role for gene dos- age in cyst initiation in polycystic kidney disease. Kidney Int. 2009 Apr;75(8):848-55.

15 Bergmann C, von Bothmer J, Ortiz Brüchle N, Venghaus A, Frank V, Fehrenbach H, et al. Mutations in multiple PKD genes may explain early and severe polycystic kidney disease. J Am Soc Nephrol. 2011 Nov;22(11):2047-56.

16 Zerres K, Rudnik-Schöneborn S, Senderek J, Eggermann T, Bergmann C. Autosomal recessive polycystic kidney disease (ARPKD). J Nephrol. 2003 May-Jun;16(3):453-8.

$17 \mathrm{Lu} \mathrm{H}$, Galeano MCR, Ott E, Kaeslin G, Kausalya PJ, Kramer C, et al. Mutations in DZIP1L, which encodes a ciliary-transitionzone protein, cause autosomal recessive polycystic kidney disease. Nat Genet. 2017 Jul; 49(7):1025-34.

18 Guay-Woodford LM, Desmond RA. Autosomal recessive polycystic kidney disease: the clinical experience in North America. Pediatrics. 2003 May;111(5 Pt 1):1072-80.

19 Burgmaier K, Kilian S, Bammens B, Benzing $\mathrm{T}$, Billing $\mathrm{H}$, Büscher A, et al. Clinical courses and complications of young adults with autosomal recessive polycystic kidney disease (ARPKD). Sci Rep. 2019 May 28;9(1):7919.

20 O’Toole JF, Otto EA, Hoefele J, Helou J, Hildebrandt F. Mutational analysis in 119 families with nephronophthisis. Pediatr Nephrol. 2007 Mar;22(3):366-70.

21 Luo F, Tao YH. Nephronophthisis: a review of genotype-phenotype correlation. Nephrology. 2018 Oct;23(10):904-11.

22 Sahay M. Congenital anomalies of kidney and urinary tract (CAKUT). Clin Queries: Nephrol. 2013;2(4):156-65.

23 Gendreau S, Servais A, Cohen C. The case atrophic kidney and ocular abnormalities. Kidney Int. 2020 Oct;98(4):1059-60.

24 Okumura T, Furuichi K, Higashide T, Sakurai $\mathrm{M}$, Hashimoto S, Shinozaki Y, et al. Association of PAX2 and other gene mutations with the clinical manifestations of renal coloboma syndrome. Plos One. 2015 Nov 16;10(11):e0142843.

25 Mironovich OL, Bliznetz EA, Markova TG, Alekseeva NN, Golybeva TI, Ryzhkova OP, et al. Molecular genetic causes and clinical description of branchio-oto-renal syndrome. Russ J Genet. 2019 May;55(5):630-8.

26 Lindau TA, Cardoso AC, Rossi NF, Giacheti CM. Anatomical changes and audiological profile in branchio-oto-renal syndrome: a literature review. Int Arch Otorhinolaryngol. 2014 Jan;18(1):68-76.

27 Chang EH, Menezes M, Meyer NC, Cucci RA, Vervoort VS, Schwartz CE, et al. Branchiooto-renal syndrome: the mutation spectrum in EYA1 and its phenotypic consequences. Hum Mutat. 2004 Jun;23(6):582-9.

28 Kohlhase J. SALL1 mutations in TownesBrocks syndrome and related disorders. Hum Mutat. 2000 Dec;16(6):460-6.

29 Miller EM, Hopkin R, Bao L, Ware SM. Implications for genotype-phenotype predictions in Townes-Brocks syndrome: case report of a novel SALL1 deletion and review of the literature. Am J Med Genet A. 2012 Mar; $158 \mathrm{~A}(3): 533-40$.

30 Ha TS. Genetics of hereditary nephrotic syndrome: a clinical review. Korean J Pediatr. 2017 Mar;60(3):55-63.

31 Rood IM, Deegens JK, Wetzels JF. Genetic causes of focal segmental glomerulosclerosis: implications for clinical practice. Nephrol Dial Transplant. 2012 Mar;27(3):882-90.

32 Lin BL, Matera D, Doerner JF, Zheng N, Del Camino D, Mishra S, et al. In vivo selective inhibition of TRPC6 by antagonist BI 749327 ameliorates fibrosis and dysfunction in cardiac and renal disease. Proc Natl Acad Sci USA. 2019 May 14;116(20):10156-61.

33 Hudson BG, Tryggvason K, Sundaramoorthy M, Neilson EG. Alport's syndrome, goodpasture's syndrome, and type IV collagen. N Engl J Med. 2003 Jun 19;348(25):2543-56.

34 Nozu K, Nakanishi K, Abe Y, Udagawa T, Okada S, Okamoto T, et al. A review of clinical characteristics and genetic backgrounds in Alport syndrome. Clin Exp Nephrol. 2019 Feb;23(2):158-68.

35 Warady BA, Agarwal R, Bangalore S, Chapman A, Levin A, Stenvinkel P, et al. Alport syndrome classification and management. Kidney Med. 2020 Sep-Oct;2(5):639-49.

36 Mencarelli MA, Heidet L, Storey H, van Geel M, Knebelmann B, Fallerini C, et al. Evidence of digenic inheritance in Alport syndrome. J Med Genet. 2015 Mar;52(3):163-74.

37 Wang Y, Sivakumar V, Mohammad M, Colville D, Storey H, Flinter F, et al. Clinical and genetic features in autosomal recessive and X-linked Alport syndrome. Pediatr Nephrol. 2014 Mar;29(3):391-6.

38 Storey H, Savige J, Sivakumar V, Abbs S, Flinter FA. COL4A3/COL4A4 mutations and features in individuals with autosomal recessive Alport syndrome. J Am Soc Nephrol. 2013 Dec;24(12):1945-54.

39 Savige J, Storey H, Il Cheong H, Gyung Kang $\mathrm{H}$, Park E, Hilbert P, et al. X-linked and autosomal recessive Alport syndrome: pathogenic variant features and further genotype-phenotype correlations. Plos One. 2016;11(9): e0161802. 
40 Soriano JR. Renal hereditary tubulopathies: from the clinic to the molecular biology. $\mathrm{Ne}$ frologia. 2003;23:71-83.

41 Ashton EJ, Legrand A, Benoit V, Roncelin I, Venisse A, Zennaro MC, et al. Simultaneous sequencing of 37 genes identified causative mutations in the majority of children with renal tubulopathies. Kidney Int. 2018 Apr; 93(4):961-7.

42 Iancu D, Ashton E. Inherited renal tubulopathies-challenges and controversies. Genes. 2020 Mar 5;11(3):277.

43 Batlle D, Haque SK. Genetic causes and mechanisms of distal renal tubular acidosis Nephrol Dial Transplant. 2012 Oct;27(10): 3691-704.

44 Benito MH, Garcia-Gonzalez MA, Recio MV, Ordas AM, Martinez RC, Gomez MAR, et al. The need for genetic study to diagnose some cases of distal renal tubular acidosis. Nefrologia. 2016 Sep-Oct;36(5):552-55.

45 Zheng W, Wu Y, Huang W. A case report on ATP6V0A4 gene mutation: forecast of familial deafness by genetic investigation in a patient with autosomal recessive distal renal tubular acidosis. Trop J Pharm Res. 2016 Nov; 15(11):2527-30.

46 Zhou F, Mao J, Ye Q, Zhu X, Zhang Y, Ye Y, et al. Clinical features and genetic findings in Chinese children with distal renal tubular acidosis. Int J Clin Exp Pathol. 2018;11(7) 3523-32.

47 Klootwijk ED, Reichold M, Unwin RJ, Kleta R, Warth R, Bockenhauer D. Renal Fanconi syndrome: taking a proximal look at the nephron. Nephrol Dial Transplant. 2015 Sep 30(9):1456-60.

48 van der Wijst J, Belge $\mathrm{H}$, Bindels RJM, Devuyst O. Learning physiology from inherited kidney disorders. Physiol Rev. 2019 Jul 1;99(3): 1575-653.

49 Yamamoto K, Cox JP, Friedrich T, Christie PT, Bald M, Houtman PN, et al. Characterization of renal chloride channel (CLCN5) mutations in Dent's disease. J Am Soc Nephrol. 2000 Aug;11(8):1460-8.

50 Bokenkamp A, Bockenhauer D, Cheong HI, Hoppe B, Tasic V, Unwin R, et al. Dent-2 disease: a mild variant of Lowe syndrome. J Pediatr. 2009 Jul;155(1):94-9.

51 Gianesello L, Del Prete D, Ceol M, Priante G, Calò LA, Anglani F. From protein uptake to dent disease: an overview of the CLCN5 gene. Gene. 2020 Jul 15;747:144662.
52 Attard M, Jean G, Forestier L, Cherqui S, Broyer M, van't Hoff W, et al. Mutations in the cystinosis, CTNS, gene: correlation between genotype and phenotype. Hum $\mathrm{Mol}$ Genet. 1999 Dec;8(13):2507-14.

53 Zarkhin V, Lovelace P, Maecker H, Sarwal MM. Characterization of novel lysosomal genes for immune regulation and spermatogenesis in nephropathic cystinosis. Immune response in patients with nephropathic cystinosis. Pediatr Nephrol. 2010 Oct;25(10): 2204-5.

54 Thoene J, Lemons R, Anikster Y, Mullet J, Paelicke K, Lucero C, et al. Mutations of CTNS causing intermediate cystinosis. Mol Genet Metab. 1999 Aug;67(4):283-93.

55 Browning AC, Figueiredo GS, Baylis O, Montgomery E, Beesley C, Molinari E, et al. A case of ocular cystinosis associated with two potentially severe CTNS mutations. Ophthalmic Genet. 2019 Apr;40(2):157-60.

56 Germain DP, Benistan K, Angelova L. Xlinked inheritance and its implication in the diagnosis and management of female patients in Fabry disease. Rev Med Interne. 2010 Dec; 31(Suppl 2):S209-13.

57 Choi JH, Lee BH, Heo SH, Kim GH, Kim YM, Kim DS, et al. Clinical characteristics and mutation spectrum of GLA in Korean patients with Fabry disease by a nationwide survey: underdiagnosis of late-onset phenotype. Medicine. 2017 Jul;96(29):e7387.

58 Hughes D, Goker-Alpan O, Ganesh J, Lau H, Foo CWP, Souberbielle B, et al. A phase I/II multicenter gene therapy clinical study for Fabry disease. Mol Genet Metab. 2020 Feb; 129(2):S77

59 Hughes DA, Patel N, Kinch R, Dronfield L, Short G, Sheridan R, et al. First-in-human study of a liver-directed AAV gene therapy (FLT190) in Fabry disease. Mol Genet Metab. 2020 Feb;129(2):S77-8.

60 Sezerman OU, Ulgen E, Seymen N, Durasi IM. Bioinformatics workflows for genomic variant discovery interpretation and prioritization. In: Samadikuchaksaraei A, Seifi M, editors. Bioinformatics tools for detection and clinical interpretation of genomic variations: IntechOpen; 2019

61 Reilly DF, Breyer MD. The use of genomics to drive kidney disease drug discovery and development. Clin J Am Soc Nephrol. 2020 Sep 7:15(9):1342-51.

62 Armstrong ME, Thomas CP. Diagnosis of monogenic chronic kidney diseases. Curr Opin Nephrol Hypertens. 2019 Mar;28(2): 183-94.
63 Cruz NM, Freedman BS. CRISPR gene editing in the kidney. Am J Kidney Dis. 2018 Jun; 71(6):874-83.

64 Richards S, Aziz N, Bale S, Bick D, Das S, Gastier-Foster J, et al. Standards and guidelines for the interpretation of sequence variants: a joint consensus recommendation of the American college of medical genetics and genomics and the association for molecular pathology. Genet Med. 2015 May;17(5):40524.

65 Katsonis P, Koire A, Wilson SJ, Hsu TK, Lua RC, Wilkins AD, et al. Single nucleotide variations: biological impact and theoretical interpretation. Protein Sci. 2014 Dec;23(12): 1650-66.

66 Kearney HM, Thorland EC, Brown KK, Quintero-Rivera F, South ST; Working Group Of The American College Of Medical Genetics Laboratory Quality Assurance Committee. American College of medical genetics standards and guidelines for interpretation and reporting of postnatal constitutional copy number variants. Genet Med. 2011 Jul;13(7): 680-5.

67 Riggs ER, Andersen EF, Cherry AM, Kantarci S, Kearney H, Patel A, et al. Technical standards for the interpretation and reporting of constitutional copy-number variants: a joint consensus recommendation of the American college of medical genetics and genomics (ACMG) and the clinical genome resource (ClinGen). Genet Med. 2020 Feb;22(2):24557.

68 Bredrup C, Saunier S, Oud MM, Fiskerstrand T, Hoischen A, Brackman D, et al. Ciliopathies with skeletal anomalies and renal insufficiency due to mutations in the IFT-A Gene WDR19. Am J Hum Genet. 2011 Nov 11; 89(5):634-43.

69 Savige J, Ariani F, Mari F, Bruttini M, Renieri $\mathrm{A}$, Gross $\mathrm{O}$, et al. Expert consensus guidelines for the genetic diagnosis of Alport syndrome. Pediatr Nephrol. 2019 Jul;34(7):1175-89.

70 Martin P, Heiskari N, Zhou J, Leinonen A, Tumelius T, Hertz JM, et al. High mutation detection rate in the COL4A 5 collagen gene in suspected Alport syndrome using PCR and direct DNA sequencing. J Am Soc Nephrol. 1998 Dec;9(12):2291-301.

71 Kashtan CE, Gross O. Clinical practice recommendations for the diagnosis and management of Alport syndrome in children, adolescents, and young adults-an update for 2020 Pediatr Nephrol. 2021 Mar;36(3):711-9. 